\title{
CENTRAL ZERO DIVISORS IN GROUP ALGEBRAS
}

\author{
MARTHA K. SMITH
}

\begin{abstract}
A central element of the complex group algebra $\mathrm{C} G$ which is a zero divisor in the $W^{*}$ group algebra $W(G)$ is also a zero divisor in $\mathbf{C} G$. As a corollary, if $K$ is a field of characteristic zero, $G$ is a group, $A$ is an abelian normal subgroup of $G$, and $R$ is the Ore localization of $K G$ obtained by inverting all nonzero elements of $K A$, then all matrix rings over $R$ are directly finite and $R$ has the invariant basis property.
\end{abstract}

The reader is referred to $[\mathbf{1}]$ and the references therein for terminology not explained here.

THEOREM. If $z$ is a central element of $\mathbf{C} G$ which is a zero divisor in $W(G)$, then $z$ is a zero divisor in $\mathbf{C} G$.

Since a zero divisor in a group algebra must, in fact, be a zero divisor in the group algebra of its support group, and similarly for the corresponding $W^{*}$ algebras (cf. the reductions in the proof of the theorem), we obtain

COROLLARY 1. If $H$ is a subgroup of $G$ and $z$ is a central element of $\mathbf{C H}$ which is a zero divisor in $W(G)$, then $z$ is a zero divisor in $\mathbf{C H}$.

Since the group algebra of a free abelian group has no nonzero zero divisors, we also have

COROLlARY 2. If $A$ is a nontrivial, torsion-free, abelian subgroup of $G$, then every nonzero element of $\mathbf{C} A$ is a nonzero divisor in $W(G)$.

The methods of $[\mathbf{1}]$ then apply to give

COROLLARY 3. Let $K$ be a commutative integral domain of characteristic zero, $G$ a group, $A$ an abelian normal subgroup of $G$, and $R=(K G)\left[Y^{-1}\right]$, where $Y=$ $K A-\{0\}$. Then all matrix rings $M_{n}(R)$ are directly finite, and $R$ satisfies the invariant basis property.

PROOF OF THEOREM. Elements of $W(G)$ may be expressed in the form $a=$ $\sum_{g \in G} a_{g} g$. Multiplication is convolution: if $b=\sum_{g \in G} b_{g} g$, then

$$
a b=\sum_{\sigma \in G}\left(\sum_{g \in G} a_{g} b_{g^{-1} \sigma}\right) \sigma .
$$

Such a sum $a$ is in $W(G)$ if and only if $a b \in L^{2}(G)$ for all $b \in L^{2}(G)$, where $b \in L^{2}(G)$ is identified with $\sum b(g) g$ and $a b$ is defined as above. The involution on $W(G)$ is given by $a^{*}=\sum \bar{a}_{g} g^{-1}$.

Received by the editors May 31, 1983 and, in revised form, September 19, 1983.

1980 Mathematics Subject Classification. Primary 16A27, 46L99, 16A08, 43A25. 
Let $a=\sum a_{g} g \in W(G)$ be such that $z a=0$. Without loss of generality, $a_{1} \neq 0$. (1 will denote the identity of every group appearing.) Let $H$ be the subgroup of $G$ generated by the support of $z$. It then follows from the above remarks and grouping by cosets that $z a^{\prime}=0$ where $0 \neq a^{\prime}=\sum_{g \in H} a_{g} g \in W(H)$.

Since $z$ is central, $H$ is an FC group: every element has only finitely many conjugates. Thus it suffices to consider the case when $G$ is a finitely generated FC group. Such groups have the property that $G^{+}=\{g \in G \mid g$ has finite order $\}$ is a finite normal subgroup such that $G / G^{+}$is torsion-free abelian [2, Lemma 4.1.5(iii)].

Let $\bar{g}_{1}, \ldots, \bar{g}_{n}$ be a fixed set of free abelian generators for $G / G^{+}$. Let $T=\{\alpha \in$ C ||$\alpha \mid=1\}$, the circle group. Each $\gamma=\left(\gamma_{1}, \ldots, \gamma_{n}\right) \in T^{n}$ defines a homomorphism $\gamma: G / G^{+} \rightarrow \mathbf{C}^{*}$ by $\gamma\left(\bar{g}_{1}^{\nu_{1}} \cdots \bar{g}_{n}^{\nu_{n}}\right)=\gamma_{1}^{\nu_{1}} \cdots \gamma_{n}^{\nu_{n}}$. This lifts to a homomorphism $G \rightarrow \mathbf{C}^{*}$ which we also denote by $\gamma$. This in turn defines an automorphism of $W(G)$ sending $a=\sum a_{g} g$ to $a^{\gamma}=\sum a_{g} \gamma(g) g$. (This is a group action of $T^{n}$ on $W(G)$.)

Since $z$ is a zero divisor in $W(G), z e=0$ for some nonzero projection $e=e^{*}=$ $e^{2} \in W(G)$. Since $z$ is central, $e$ is central.

LEMMA 1. There is an $\varepsilon>0$ such that ee $e^{\gamma} \neq 0$ whenever $\gamma \in T^{n}$ satisfies $|\gamma-1|<\varepsilon .\left(||\right.$ denotes the usual norm in $\mathbf{C}^{n}:|\gamma|=\left(\sum\left|\gamma_{i}\right|^{2}\right)^{1 / 2}$.)

ProOF. Let $e=\sum e_{g} g$. Then the coefficient of the identity in $e e^{\gamma}$ is

$$
\left(e e^{\gamma}\right)_{1}=\sum_{g} e_{g^{-1}} \gamma(g) e_{g}=\sum \gamma(g)\left|e_{g}\right|^{2} .
$$

It is enough to produce $\varepsilon$ so that this is nonzero whenever $|\gamma-1|<\varepsilon$. Now

$$
e_{1}=\sum_{g}\left|e_{g}\right|^{2}>0 \text {. }
$$

Since this converges, there is a finite subset $S$ of $G$ such that

$$
\sum_{g \notin S}\left|e_{g}\right|^{2}<\frac{e_{1}}{3}
$$

Since each $|\gamma(g)|=1$,

$$
\left.\left.\left|\sum_{g \notin S} \gamma(g)\right| e_{g}\right|^{2}\left|\leq \sum_{g \notin S}\right| e_{g}\right|^{2}<\frac{e_{1}}{3}
$$

Now

$$
\left|e_{1}-\left(e e^{\gamma}\right)_{1}\right| \leq \sum_{g \in S}|1-\gamma(g)|\left|e_{g}\right|^{2}+\sum_{g \notin S}\left|e_{g}\right|^{2}+\sum_{g \notin S} \gamma(g)\left|e_{g}\right|^{2},
$$

so it is enough to find $\varepsilon$ so that

$$
|1-\gamma(g)|<\frac{e_{1}}{3|S|\left|e_{g}\right|^{2}}
$$

for all $g \in S$. If the image of $g$ in $G / G^{+}$is $\bar{g}_{1}^{\nu_{1}} \cdots \bar{g}_{n}^{\nu_{1}}$, then $\gamma(g)=\gamma_{1}^{\nu_{1}} \cdots \gamma_{n}^{\nu_{n}}$, which is a continuous function of $\gamma$. Hence, since $S$ is finite, the desired $\varepsilon$ exists. 
LEMMA 2. Let $\bar{x}_{1}, \ldots, \bar{x}_{m}$ be distinct elements of $G / G^{+}$. Given $\varepsilon>0$, there exist $\gamma^{(1)}, \ldots, \gamma^{(m)} \in T^{n}$ with $\left|1-\gamma^{(i)}\right|<\varepsilon, i=1,2, \ldots, m$, such that $\operatorname{det}\left(\gamma^{(i)}\left(\bar{x}_{j}\right)\right) \neq$ 0 .

PROOF. The induction on $m$ as in [2, Lemma 4.3.3] will apply if we can show that given $\varepsilon>0$ and $\alpha_{1}, \ldots, \alpha_{m} \in \mathbf{C}$, not all zero, there exists $\gamma$ with $|1-\gamma|<\varepsilon$ such that $\alpha_{1} \gamma\left(\bar{x}_{1}\right)+\cdots+\alpha_{m} \gamma\left(\bar{x}_{m}\right) \neq 0$. This just amounts to showing that a nonzero polynomial in $\gamma_{1}, \ldots, \gamma_{n}$ takes on a nonzero value in every neighborhood of 1 in $T^{n}$. This follows easily by a similar induction on $n$.

Continuing the proof of the Theorem, let $z=z_{1} x_{1}+\cdots+z_{m} x_{m}$ where $z_{i} \in$ $\mathrm{C}^{+}$and the $x_{i}$ are in distinct cosets of $G$ modulo $G^{+}$. Applying Lemma 1 inductively (recalling that $e$, hence every $e^{\gamma}$, is central), there exists $\varepsilon>0$ such that $e^{\gamma^{(1)}} e^{\gamma^{(2)}} \cdots e^{\gamma^{(m)}} \neq 0$ whenever $\left|1-\gamma^{(i)}\right|<\varepsilon, i=1,2, \ldots, m$. Therefore by Lemma 2 , there exist $\gamma^{(1)}, \ldots, \gamma^{(m)} \in T$ such that $e_{0}=e^{\gamma^{(1)}} e^{\gamma^{(2)}} \cdots e^{\gamma^{(m)}} \neq 0$ and $\operatorname{det}\left(\gamma^{(i)}\left(\bar{x}_{j}\right)\right) \neq 0$, where $\bar{x}_{j}$ is the coset of $x_{j} \bmod G^{+}$. For any $\gamma, z^{\gamma}$ is central and $z^{\gamma} e^{\gamma}=0$. Therefore $e_{0} z^{\gamma^{(i)}}=z^{\gamma^{(i)}} e_{0}=0, i=1,2, \ldots, m$. Since $z^{\gamma^{(i)}}=\gamma^{(i)}\left(\bar{x}_{1}\right) z_{1} x_{1}+\cdots+\gamma^{(i)}\left(\bar{x}_{m}\right) z_{m} x_{m}$ and $\operatorname{det}\left(\gamma^{(i)}\left(\bar{x}_{j}\right)\right) \neq 0$, it follows that $e_{0} z_{i} x_{i}=0$, hence $e_{0} z_{i}=0, i=1,2, \ldots, m$. As before, this implies there is a nonzero $c \in W(A)$, where $A$ is the subgroup generated by the supports of $z_{1}, z_{2}, \ldots, z_{m}$, with $c z_{i}=0, i=1,2, \ldots, m$. But $A \subset G^{+}$is finite, so $W(A)=\mathbf{C} A \subseteq \mathbf{C} G$. Thus $0 \neq c \in \mathbf{C} G$ and $c z=0$.

REMARKS. 1. If $G$ is finitely generated free abelian, the theorem may be proved via Fourier transforms. Briefly, a suitable version of the Plancherel Theorem says that (i) the idempotent $e$ acting on $L^{2}(G)$ corresponds to the operator in $L^{2}(\hat{G})=$ $L^{2}\left(T^{n}\right)$ given by multiplication by the characteristic function $\chi_{A}$ of some set $A$, and (ii) $z=\sum \varsigma_{g} g$ corresponds to the function $\hat{z}(\gamma)=\sum \varsigma_{i} \gamma(g)$. The equation $z e=0$ translates to $\hat{z} \chi_{A}=0$, which implies $\hat{z}(\gamma)=0$ for all $\gamma \in A$. Since $z$ is a finite sum, $A$ must have measure zero, forcing $e=0$. The more algebraic proof given above was motivated by this Fourier transform argument as follows. If $A$ has positive measure, its intersection with a translate by $\gamma$ sufficiently close to 1 must intersect $A$ in a set of positive measure. The characteristic function of the translate corresponds to $e^{\gamma}$; the characteristic function of the intersection corresponds to $e e^{\gamma}$.

2. The referee has pointed out that it is, in fact, possible to reduce to the case of a finitely-generated, torsion-free, abelian group as follows. The finitely-generated FC group has a finitely-generated, torsion-free, central subgroup $N$ of finite index. Letting $Y=K N-\{0\}, R=(H K)\left[Y^{-1}\right]$ is artinian. Thus if $z$ is not a zero divisor in $R, z$ has an inverse $x z_{0}^{-1}$ in $R$, where $z_{0} \in Y$. Since $z$ is a zero divisor in $W(G)$, $z_{0}$ is a zero divisor in $W(G)$, hence in $W(N)$. This permits a slight simplification in the notation of the remainder of the proof.

3. We have learned since submitting this paper for publication that S. Rosset has independently given a proof (using trigonometric polynomials) of Corollary 3.

\section{REFERENCES}

1. K. R. Goodearl, Localized group rings, the invariant basis property and Euler characteristics, Proc. Amer. Math. Soc. 91 (1984), 523-528.

2. D. S. Passman, The algebraic structure of group rings, Wiley, New York, 1977.

Department of Mathematics, University of Texas, Austin, Texas 78712 